\section{Plaque radiotherapy treatment with ruthenium-106 for iris malignant melanoma}

M Tsimpida, J Hungerford, A Arora and V Cohen

\begin{abstract}
Purpose To report the results of ruthenium106 plaque radiotherapy for iris malignant melanoma.

Methods A retrospective study of 15 patients with pure iris melanoma treated with ruthenium-106 plaque radiotherapy from June 1998 to June 2006. The main outcome measures were tumour control and ocular complications. Results Of the 15 patients, 8 had biopsyproven melanoma (6 incisional and 2 excisional biopsies). In the remaining seven patients enlargement of the lesion was documented. The median follow-up was 96 months (ranging from $\mathbf{1 4}$ months to $\mathbf{1 2}$ years). Common radiation-related complications included cataract in $9(60 \%)$ patients, dry eyes in $3(20 \%)$ patients and elevated intraocular pressure in $4(27 \%)$ patients. Vision was preserved in $80 \%$ of patients. Local tumour control was obtained in all patients. Conclusions Ruthenium-106 plaque radiotherapy is an effective treatment for primary malignant iris melanoma, resulting in excellent local control with preservation of vision. Main complications included cataract, dry eyes, and glaucoma.

Eye (2011) 25, 1607-1611; doi:10.1038/eye.2011.222; published online 23 September 2011
\end{abstract}

Keywords: iris melanoma; ruthenium-106 plaque radiotherapy; biopsy

\section{Introduction}

Iris melanoma is uncommon, comprising 2-3\% of all uveal melanomas. ${ }^{1}$ As only about $5 \%$ of untreated suspicious melanocytic lesions show growth in the first 5 years after detection, ${ }^{2,3}$ the majority of localized melanocytic iris lesions do not require immediate surgical excision, and management primarily involves observation for documented growth. Other clinical features that are suspicious for melanoma include basal diameter greater than $3 \mathrm{~mm}$, abnormal vasculature, presence of pigment dispersion, satellite lesions, and tumour-related symptoms. ${ }^{4,5}$ In cases that are highly suspicious for malignancy, incisional biopsy or fine needle aspiration biopsy can be performed for tissue diagnosis.

With regard to management, localized iris melanomas are treated with local excision using the techniques of iridectomy, iridocyclectomy, or iridogoniocyclectomy. ${ }^{2}$ In cases of unresectable iris melanoma or in tumours with extensive seeding, plaque radiotherapy, proton beam irradiation, or enucleation can be used. ${ }^{6-8}$ Enucleation is the treatment of choice in cases of diffuse melanoma where more than half of the iris and trabecular meshwork are invaded by tumour or when there is uncontrollable glaucoma.

The present study was undertaken to evaluate the use of plaque radiotherapy with ruthenium-106 for the treatment of iris melanoma. The outcomes measured were local tumour control, patient survival, and the type and incidence of ocular complications.

In the discussion, a comparison is made with other treatment modalities for iris melanoma, including radioactive plaques of different isotopes (iodine-125), proton-beam irradiation, and local resection.

\section{Materials and methods}

A retrospective review of the records of all patients treated with ruthenium-106 plaque radiotherapy in the ocular oncology service at St Bartholomew's Hospital and Moorfields Eye Hospital between June 1998 and June 2006
Department of Ophthalmology,

St Bartholomew's Hospital and Moorfields Eye Hospital, London, UK

Correspondence: M Tsimpida, Department of Ophthalmology, St Bartholomew's Hospital and Moorfields Eye Hospital, West Smithfield, 162 City Road, London EC 1V 2PD, UK Tel: + 44 (0)203 465 5907; Fax: + 44 (0)203 4655936 . E-mail: maria.tsimpida@ bartsandthelondon.nhs.uk

Received: 4 April 2011 Accepted in revised form: 6 June 2011

Published online:

23 September 2011

This study has been accepted as an oral presentation in the Joint Congress of SOE AAO, 4-7 June 2011, Geneva Switzerland and the Ophthalmic Oncology Group Meeting, Copenhagen, 15-17 April 2011. 
identified patients with the diagnosis of iris malignant melanoma. The criteria for use of plaque radiotherapy included documented growth and histopathology results confirming malignancy after incisional/or excisional biopsy (performed by VC and JH). In this analysis, only tumours arising from the iris were assessed, termed pure iris melanoma. Those with ciliary body extension or lesions arising primarily from the ciliary body were excluded. This was determined clinically by transpupillary transillumination and anterior segment ultrasound. Informed consent was obtained after discussion of the findings, potential benefits, and complications. Information regarding age at treatment, sex, and laterality was collected. The pre-treatment ocular data included visual acuity and intraocular pressure at presentation, iris colour, tumour quadrant (superior, temporal, inferior, and nasal), tumour configuration (nodular and diffuse), tumour base (in clock hours extension), presence of infiltration of the angle, and extraocular extension. The radiation parameters included plaque shape (round and notched), plaque diameter (15 and $20 \mathrm{~mm}$ ), prescribed dose (Gy) and depth $(\mathrm{mm})$, radiation dose to tumour apex and sclera $(\mathrm{Gy})$, and radiation rate $(\mathrm{Gy} / \mathrm{h})$. Patients were reviewed at regular intervals (by $\mathrm{VC}$ and $\mathrm{JH}$ ) with documentation of visual acuity, intraocular pressure, slit-lamp assessment, and anterior segment photography. Loss to follow-up was recorded.

Statistical analysis was performed using Kaplan-Meier estimates for the development of cataract following plaque radiotherapy treatment. Other results are expressed as percentages, means, medians, and range.

\section{Results}

A total of 15 patients with primary iris melanoma treated with ruthenium-106 plaque radiotherapy were identified. The demographic characteristics of the patients and the pre-treatment features are described in Tables 1 and 2, respectively. In 12 patients the mean intraocular pressure at presentation was $17 \mathrm{~mm} \mathrm{Hg}$ (ranging from 10 to $21 \mathrm{~mm} \mathrm{Hg}$ ). Three patients presented with intraocular pressures of 33,38 , and $40 \mathrm{~mm} \mathrm{Hg}$, respectively. The mean number of clock hours of tumour diameter was 2 (ranging from 1 to 3 clock hours). None of the melanomas showed ciliary body involvement or extraocular extension. The tumour location is described in Table 3.

A total of eight patients presented with a suspicious, elevated lesion that warranted biopsy to confirm the diagnosis. In six of these patients an incisional biopsy was performed and two patients had excisional biopsy of the iris lesion. The histopathological features of the excised iris lesions are described in Table 4.
Table 1 Demographics

\begin{tabular}{lc}
\hline Age at treatment (mean) & $\begin{array}{c}55 \text { years (ranging from } \\
30 \text { to } 81 \text { years) }\end{array}$ \\
Sex & $\mathrm{M} / \mathrm{F}=9 / 6$ \\
Laterality & $\mathrm{RE} / \mathrm{LE}=7 / 8$ \\
Iris colour & \\
Grey & \\
Blue & $13(86 \%)$ \\
Brown & $1(7 \%)$ \\
\hline
\end{tabular}

Values are given as number or ratio.

Table 2 Pre-treatment features

\begin{tabular}{lc}
\hline Feature & Number (\%) \\
\hline Snellen visual acuity & \\
$6 / 6$ & $12(80)$ \\
$6 / 9$ & $2(13)$ \\
$6 / 36$ & $1(7)$ \\
IOP & \\
IOP $\leq 16 \mathrm{~mm} \mathrm{Hg}$ & $4(27)$ \\
IOP $=17-21 \mathrm{~mm} \mathrm{Hg}$ & $8(53)$ \\
IOP $\geq 30$ mm Hg & $3(20)$ \\
& \\
Gonioscopy & \\
No angle involvement & $11(73)$ \\
No angle involvement with increased & $5(33)$ \\
angle pigmentation & \\
Local invasion of angle & $4(27)$ \\
Local invasion of angle with increased & $1(7)$ \\
angle pigmentation & \\
Tumour appearance & \\
Melanotic & \\
Amelanotic & $11(73)$ \\
Mixed & $1(7)$ \\
Tapioca & $3(20)$ \\
\hline
\end{tabular}

A 15-mm round plaque was used in $10(67 \%)$ patients, a $20-\mathrm{mm}$ round plaque in $4(27 \%)$ patients, and 1 patient had a notched $20-\mathrm{mm}$ plaque placed. The prescribed radiation dose was $80 \mathrm{~Gy}$ in all cases, at a mean depth of $4 \mathrm{~mm}$ (ranging from 3 to $5 \mathrm{~mm}$ ). The mean radiation rate was $1.3 \mathrm{~Gy} / \mathrm{h}$ (ranging from 0.8 to $2.6 \mathrm{~Gy} / \mathrm{h}$ ). The radiation dose to the tumour apex ranged from 106 to 119 Gy (mean 97 Gy) and the scleral dose was 173 Gy (ranging from 157 to $326 \mathrm{~Gy}$ ).

The median follow-up period was 96 months (ranging from 14 months to 12 years). Tumour control was achieved in $15 / 15$ patients (100\%). No tumours required any salvage treatment as there were no local recurrences. There was no loss to follow-up. All patients were 
Table 3 Tumour location and configuration

\begin{tabular}{lc}
\hline Feature & Number (\%) \\
\hline Tumour quadrant location & \\
Superior & $6(40)$ \\
Inferior & $2(13)$ \\
Nasal & $3(20)$ \\
Inferonasal & $3(20)$ \\
Inferotemporal & $1(7)$ \\
Superotemporal & \\
Central tumour margin & \\
Pupil & $10(67)$ \\
Midzone & $3(20)$ \\
Root & $2(13)$ \\
Angle & 0 \\
Peripheral tumour margin & \\
Pupil & \\
Midzone & 0 \\
Root & 0 \\
Angle & $11(73)$ \\
Tumour configuration & $4(27)$ \\
Nodular & \\
Flat & \\
Extraocular extension & $13(87)$ \\
Ciliary body involvement & $2(13)$ \\
\hline
\end{tabular}

Table 4 Histopathology

\begin{tabular}{lc}
\hline Features on eight iris malignant melanomas & Number \\
\hline Morphology & \\
Spindle cells & 6 \\
Epithelioid cells & 1 \\
Mixed & 1 \\
Not stated & \\
& \\
Cytology & 3 \\
Nuclear pleomorphism & 2 \\
Prominent nucleoli & 3 \\
Nuclear pleomorphism and prominent nucleoli & \\
Mitotic rate & \\
Few & 4 \\
Low & 1 \\
Not stated & 3 \\
\hline
\end{tabular}

screened with biannual liver function tests and annual abdominal ultrasound. No patient developed metastasis over the period of follow-up.

Post-op transient iritis was universal after radiotherapy, lasting for 3-5 weeks following treatment and resolving with topical anti-inflammatory medications and cycloplegia. With regard to

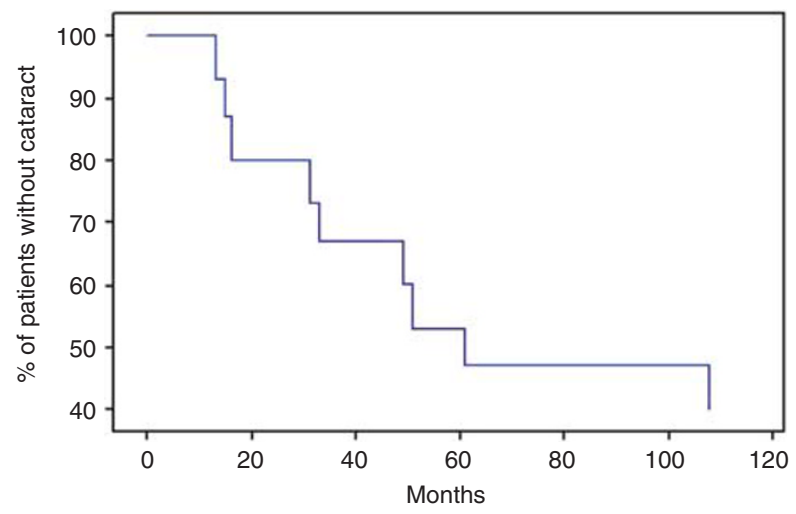

Figure 1 Kaplan-Meier estimates for the onset of cataract following plaque radiotherapy treatment.

radiation-related complications, 9/15 (60\%) of patients developed cataract. Of these, six patients had cataract extraction and intraocular lens implantation. The Kaplan-Meier estimates for the onset of cataract over the period of follow-up are displayed in Figure 1. Three patients had deterioration in vision (two of them secondary to lens opacities and in the third patient due to complicated cataract surgery).

A total of $3(20 \%)$ patients developed symptomatic dry eyes that were relieved with simple topical medications. In 1 (7\%) patient band keratopathy became evident 3 years after the plaque insertion. In $1(7 \%)$ patient thinning of the conjunctiva was noted at the site of plaque insertion 2 years post-plaque and in another patient a tenons cyst developed 5 years after the plaque placement. Moreover, in one patient significant diffuse episcleritis was evident soon after the plaque removal, which responded well to mild steroid topical therapy. There was no incidence of scleral or corneal melt.

Three patients presented with increased intraocular pressure (IOP $>30 \mathrm{~mm} \mathrm{Hg}$ ) that persisted after plaque treatment. In one of these patients pre-treatment gonioscopy showed the tumour to be invading the angle locally. In the second case the formation of peripheral anterior synechiae was evident on gonioscopy. No cause was found for the elevated IOP in the third case. Postoperatively, the IOP did not fall in any of the three patients and topical medications were required for its control. Moreover, one patient had normal IOPs on presentation but developed ocular hypertension after the plaque treatment. Gonioscopy revealed unilateral pigmentation in the angle at $360^{\circ}$. The pressure was controlled on topical medications and its increase was attributed to scatter of pigment from the iris lesion to the angle, secondary to the plaque treatment. 


\section{Discussion}

It is difficult to find a publication on iris melanoma where the angle or ciliary body is not involved. Pure iris melanomas as described in this series have the best survival outcomes. After a median follow-up of 96 months and maximum follow-up of 12 years, no patient died of metastatic disease. The pathology on these tumours demonstrates predominantly low-grade melanomas and this may help to explain the lack of local recurrence in our series. Iris melanomas do not change greatly in size or appearance following ruthenium plaque radiotherapy, which also is typical for the behaviour of low-grade (Class 1) melanomas.

The management of melanocytic lesions of the iris has become more conservative over the years. Resection techniques are reserved only for localized lesions of small basal diameter, whereas plaque radiotherapy and proton beam irradiation are used for larger iris malignant melanomas.

Local resection is still a management option for iris melanoma. However, the side effects resulting from this type of intraocular surgery may discourage the patient and the surgeon from selecting this treatment modality. One study of 51 iris malignant melanomas treated with conservative surgical treatment reported a $67 \%$ reduction in the initial visual acuity. ${ }^{9}$ In our experience, we have found that patients often complain of glare produced by correctopia and pupil irregularities following local resection of iris malignant melanoma. The best way to avoid this visual disturbance is to perform a pupiloplasty at the time of removal of the melanoma. In addition, excision of an iris malignant melanoma without adjuvant radiation carries a high risk of local recurrence of the tumour. In the same series of 51 patients treated with local resection alone, ${ }^{9} 4$ patients suffered local recurrences, whereas in our series none of the patients had a recurrence over a median follow-up period of 96 months. Compared with intraocular excision, ophthalmic plaque radiotherapy is a safer treatment modality with fewer intraocular complications, such as intraocular haemorrhage, infection, and, most importantly, tumour seeding.

Excellent tumour control (100\%) was achieved following ruthenium plaque radiotherapy in our series, in contrast to the reduced tumour control obtained with other treatment modalities. ${ }^{7,8}$

Many of the complications of ocular radiotherapy have a considerably delayed onset, often years after the initial treatment. In this series the median follow-up was long, at 96 months. Therefore, this paper is likely to capture all the side effects expected from plaque radiotherapy. The radiation dose to the fovea and the optic nerve has been proven to be minimal after plaque radiotherapy due to the long distance between the plaque radiation source and the posterior segment. ${ }^{10}$ In our series no radiation maculopathy, papillopathy, or retinopathy developed secondary to plaque radiotherapy. Although there have been concerns regarding the radiation tolerance of the cornea and sclera, in this series no visually significant corneal opacities, corneal and/or scleral melt were documented. This has also been documented following the use of radiotherapy for iris malignant melanoma with palladium-103 and iodine-125 isotopes. ${ }^{7,10}$

The incidence of elevated IOP reported after ruthenium-106 plaque radiotherapy in our series was $27 \%$ (4 patients). In 2007 Rundle et $a l^{8}$ published a review of 15 cases with iris melanoma treated with proton beam irradiation. Elevated IOP was noted in $8(53 \%)$ cases. Of these, five patients had an increased IOP pre-operatively. The IOP did not fall following proton-beam irradiation, a fact that was attributed to trabecular scarring. This is similar to our experience, as three patients in our series had persistent raised IOP following plaque radiotherapy. None of our patients developed neovascular glaucoma, although it has been reported after proton-beam and iodine-125 radiotherapy. ${ }^{7,8}$

In this series, ocular surface complications in the form of dry eyes in $20 \%$ of patients and band keratopathy in one patient were reported. Interestingly, the incidence of dry eyes following treatment with charged particles $(27 \%)$ is quite similar to the reported incidence of dry eyes in our series.

The formation of cataract following ruthenium plaque radiotherapy was documented in $60 \%$ of patients. Shields et $a l^{6,7}$ reported on the outcome of using iodine-125 plaque radiotherapy for iris melanoma in 1995 and 2003. The incidence of cataract formation was found to be $70 \%$. This is due to the different radiation properties of iodine-125 and ruthenium-106. Iodine is predominantly a $\gamma$-emitter with increased scatter and depth of penetration, resulting in an increased risk of cataract formation. Ruthenium is predominately a $\beta$-emitter with less depth of penetration, providing a higher scleral dose. This explains the conjunctival thinning in one patient in our series and necessitates the monitoring of the scleral dose.

In summary, ruthenium-106 plaque radiotherapy was found to be effective for the treatment of malignant iris melanoma. In our series of 15 patients with a maximum follow-up of 12 years the most common complication was found to be cataract formation, which is surgically reversible; hence the majority of our patients retained their pre-treatment initial visual acuity. There were no cases of tumour recurrence and the side effects were minimal, proving plaque radiotherapy with ruthenium106 to be a safe and effective treatment for iris primary malignant melanoma. 


\section{Summary}

\section{What was known before}

- The treatment options of iris malignant melanomas include local resection, plaque radiotherapy, proton-bean irradiation, and enucleation.

- There are reports in the literature that evaluate the use of plaque radiotherapy with iodine-125 and palladium-103 for the treatment of iris malignant melanoma.

What this study adds

- In this study, we evaluate the use of plaque radiotherapy with ruthenium-106 for the treatment of iris malignant melanoma.

- The main outcome measures reported are tumour control and ocular complications.

- A comparison is also made with other treatment modalities including radioactive plaques of different isotopes (iodine-125), proton-beam irradiation, and local resection.

\section{Conflict of interest}

The authors declare no conflict of interest.

\section{Acknowledgements}

We would like to thank Dr Ian Stoker, radiotherapy physicist, for his help in this paper.

\section{References}

1 Shields CL, Shields JA, Materin M, Gershenbaum E, Singh AD, Smith A. Iris melanoma: risk factors for metastasis in 169 consecutive patients. Ophthalmology 2001; 108: 172-178.

2 Shields JA, Shields CL. Melanocytic tumours of the iris stroma. Melanoma. In: Shields JA, Shields CL (eds). Intraocular Tumours. A Text and Atlas. WB Saunders: Philadelphia, 1992, pp 69-77.

3 Territo C, Shields CL, Shields JA, Augsburger JJ, Schroeder RP. Natural course of melanocytic tumours of the iris. Ophthalmology 1988; 95: 1251-1255.

4 Shields JA, Sanborn GE, Ausburger JJ. The differential diagnosis of malignant melanoma of the iris. Ophthalmology 1983; 90: 716-720.

5 Harbour JW, Ausburger JJ, Eagle RC. Initial management and follow-up of melanocytic iris tumours. Ophthalmology 1995; 12: 1987-1993.

6 Shields CL, Shields JA, De Potter P, Singh AD, Hernandez C, Brady LW. Treatment of non-resectable malignant iris tumours with custom-design plaque radiotherapy. $\mathrm{Br} \mathrm{J}$ Opthalmolo 1995; 79: 306-312.

7 Shields CL, Naseripour M, Shields JA, Freire J, Cater J. Custom designed plaque radiotherapy for non-resectable iris melanoma in 38 pts: tumour control and ocular complications. Am J Ophthalmol 2003; 135: 648-656.

8 Rundle P, Singh AD, Rennie I. Proton beam therapy for iris melanoma: a review of 15 cases. Eye 2007; 21: 79-82.

9 Conway RM, Chua WC, Qureshi C, Billson FA. Primary iris melanoma: diagnostic features and outcome of conservative surgical treatment. Br J Ophthalmol 2001; 85: 848-854.

10 Finger PT. Plaque radiation therapy for malignant melanoma of the iris and ciliary body. Am J Ophthalmol 2001; 132: 328-335. 